\title{
Bayesian Statistical Model for Imaging of Single La Vacancies in $\mathrm{LaMnO}_{3}$
}

\author{
Jie Feng ${ }^{1}$, Alexander V. Kvit ${ }^{1}$, Chenyu Zhang ${ }^{1}$, Dane Morgan ${ }^{1}$, Paul M. Voyles ${ }^{1}$ \\ ${ }^{1 .}$ Department of Materials Science and Engineering, University of Wisconsin-Madison, Madison, \\ Wisconsin, USA
}

Previous simulation studies have demonstrated that single La vacancies can be detected using high precision quantitative high-angle annular dark-field (HAADF) Z-contrast scanning transmission electron microscopy (STEM) [1] by both the reduction in scattered intensity created by the missing atom and the distortion of the surrounding atom positions [2]. We have now experimentally detected single La vacancies on nominally stoichiometric $\mathrm{LaMnO}_{3}$ films grown on $\mathrm{DyScO}_{3}$ substrate by molecular beam epitaxy with $\sim 1 \%$ control of cation stoichiometry. The vacancy depth is determined from channeling behavior of the electron probe, quantified by a Bayesian statistical model that compares experiments to a library of simulations

Figure 1 (a) is an experimental high-precision quantitative HAADF STEM image of $\mathrm{LaMnO}_{3}$ along [100]. The TEM sample thickness is $6.5 \mathrm{~nm}$. A simulated image at the same specimen thickness and collection angle is in the white dashed box. For each La-O column, we studied its visibility $(V)$ and inter-column separation changes $\left(\Delta S_{1}\right.$ and $\left.\Delta S_{2}\right)$ [2]. For column A, marked in (a), $V=10.6 \%, \Delta S_{1}=3.9 \mathrm{pm}$, and $\Delta S_{2}=$ $3.0 \mathrm{pm}$. Figure 1 (b) and (c) are simulated $V$ and simulated $\Delta S_{1}$ and $\Delta S_{2}$ for a $6.5 \mathrm{~nm}$ TEM specimen, respectively. If we consider a conservative uncertainty band twice the estimated experimental intensity uncertainty of $1 \%$, the visibility is consistent with a vacancy anywhere from 1.1 to $5.2 \mathrm{~nm}$ deep in the sample. $\Delta S_{1}$ and $\Delta S_{2}$, given an uncertainty of $1 \mathrm{pm}$, indicate the vacancy is either $0-0.8 \mathrm{~nm}$ deep or near $3.5 \mathrm{~nm}$ deep. The overlap of all three factors localizes the vacancy to 3.4 to $4.2 \mathrm{~nm}$ deep into the sample. Figure 1 (d) shows the Bayesian statistical model calculation results on column A that the probability it contains a single La vacancy is greater than $99.9 \%$, and the most probable depth (65\% probability) is 3.5 $\mathrm{nm}$ (the 7 th atomic layer).

In order to validate the Bayesian statistical model, we have done a series of phantom tests. In each test, fake experimental measurements were created by adding noise to the noiseless simulated $V, \Delta S_{1}$, and $\Delta S_{2}$ from a specific $\mathrm{LaMnO}_{3}$ model. Then we tested whether the Bayesian model correctly recovered whether or not a La vacancy was present and the depth of the vacancy if it was present. The noise was drawn from a normal distribution with mean value of 0 , standard deviation of $1 \%$ for $V$, and 1 pm for $\Delta S_{1}$ and $\Delta S_{2}$. Each test was repeated with 10000 different noise realizations.

Figure 2 is a contour plot of the phantom test results with the real vacancy depth in the model on the $x$ axis and the Bayes model result on the $y$ axis. For example, the Bayesian model predicted that there is no La vacancy in the model in all 10000 tests from the $\mathrm{LaMnO}_{3}$ model without a vacancy. For a single vacancy $\mathrm{LaMnO}_{3}$ model with the vacancy at $2.4 \mathrm{~nm}$ depth, the Bayes model found the vacancy at the correct depth in $61 \%$ tests, $38 \%$ tests had a 1-layer prediction error and only <1\% test had a 2 -layer error. If only \pm 1 layer prediction uncertainty is allowed, the precision is higher than $85 \%$ in most cases when the vacancy depth is smaller than $7 \mathrm{~nm}$. This demonstrates that the vacancy depth calculated by the Bayesian model is very reliable and the Bayesian model is able to precisely locate a single La vacancy up to $7 \mathrm{~nm}$ deep with \pm 1 layer uncertainty [3]. 


\section{References:}

[1] A. B. Yankovich et al., "Picometre-precision analysis of scanning transmission electron microscopy images of platinum nanocatalysts.," Nat. Commun., vol. 5, no. May, (2014) p. 4155.

[2] J. Feng et al, "Prospects for Detecting Single Vacancies by Quantitative Scanning Transmission Electron Microscopy," vol. 21, no. 942, (2015) pp. 1887.

[3] This work was supported by the US Department of Energy, Basic Energy Sciences, Grant DE-FG02$08 E R 46547$.
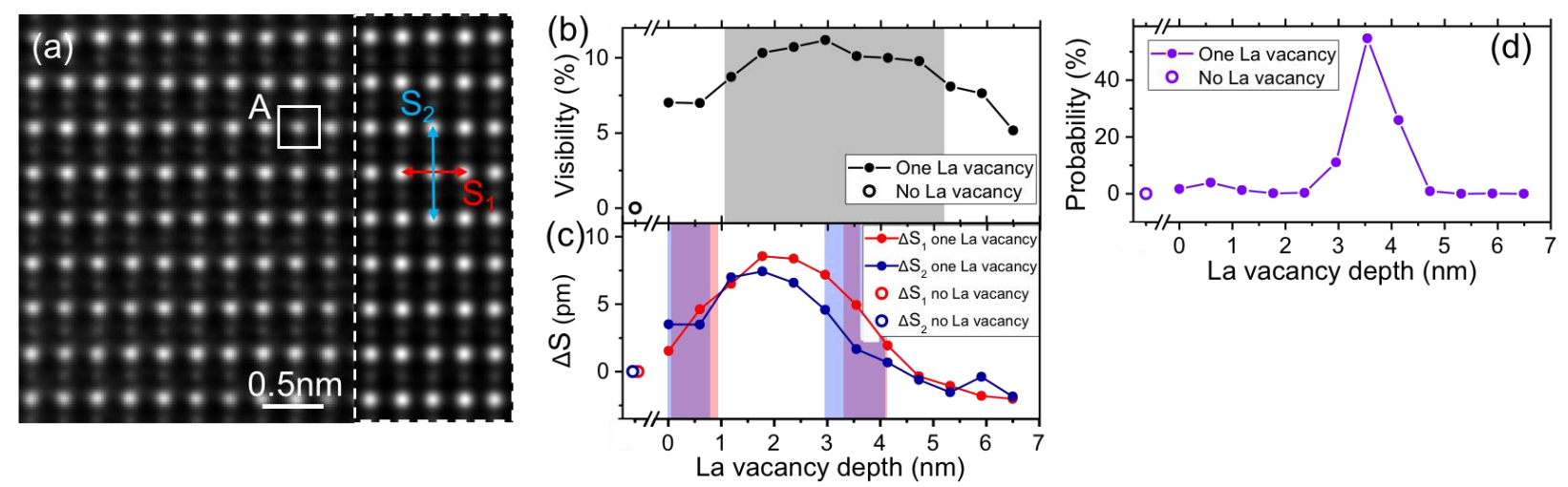

Figure 1. (a) HAADF STEM image of LaMnO3 along [100]. (b) and (c) Simulated visibility and simulated $\Delta S_{1}$ and $\Delta S_{2}$ for a TEM specimen of the same thickness. (d) Probability for the vacancy position calculated by the Bayesian model.

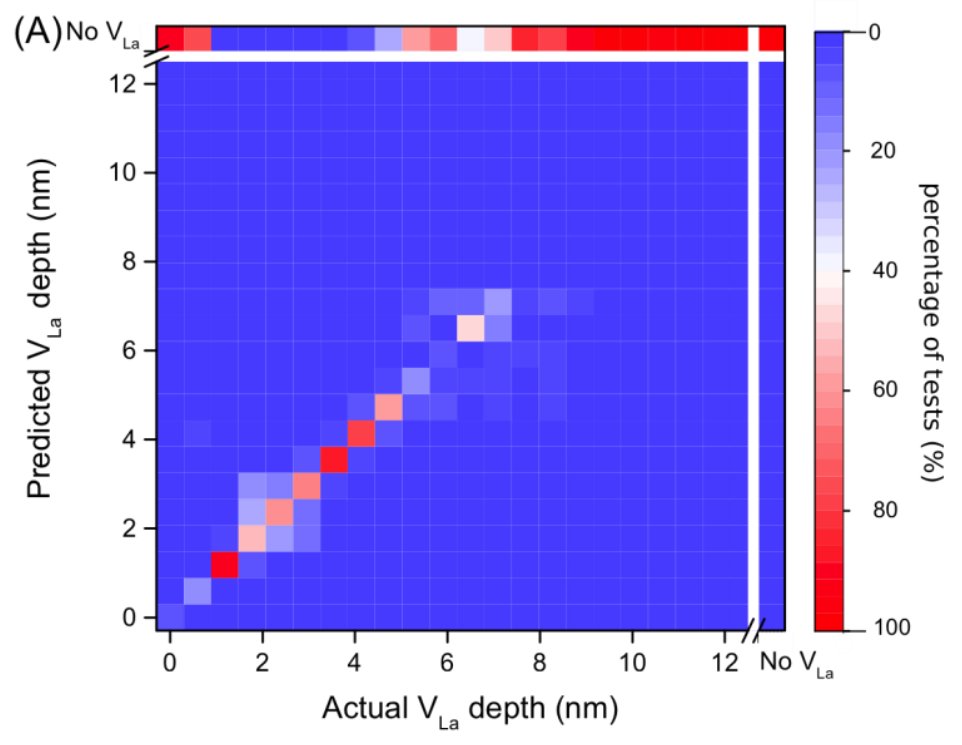

Figure 2. Phantom tests on the Bayesian statistical model on $12 \mathrm{~nm} \mathrm{LaMnO}$ model. Predicted La vacancy depth vs. the actual La vacancy depth. 